\title{
STRESS-STRAIN STATE OF ELASTIC PLATE WITH AN ARBITRARY SMOOTH NOTCH
}

\author{
Ihor KUZ*, Olga KUZ*', Heorgij SULYM***
}

\begin{abstract}
*Department of Mechanics and Mathematics, Ivan Franko National University of Lviv, 1 Universitetska Str., 79000 Lviv, Ukraine "Vyacheslav Chornovil Institute of Ecology, Nature Protection and Tourism, National University "Lviv Polytechnic", 12 Bandery Str.,79013 Lviv,Ukraine ${ }^{* * *}$ Faculty of Mechanical Engineering, Bialystok University of Technology, 45C Wiejska Str., 15-351 Bialystok, Poland
\end{abstract}

ihorkuz24@gmail.com, olyakuzon@gmail.com, sulym@pb.edu.pl

received 4 May 2015, revised 15 December 2015, accepted 16 December 2015

\begin{abstract}
The paper contains comparing calculations of the stress fields in an elastic plate with notch along the arc of a circle, ellipse or parabola obtained by analytic method based on complex Kolosov-Muskhelishvili potentials and by numerical variation-difference method. These fields differ by no more than $2 \%$, which, in particular, indicates the reliability of such numerical implementation. This discrepancy can be explained by the fact that in the analytical solution domain is unbounded, while the numerical calculation was carried out, obviously, for a finite field. The given stresses at the top of the notch along the arc of an ellipse or a parabola significantly increase with increasing of the relative depth of the notch (while increasing its depth or decreasing width).
\end{abstract}

Keywords: Semi-Plane, Plate, Notch, Variation-Difference Method, Stress Field

\section{INTRODUCTION}

Investigation of the stress-strain state of the plate structural elements weakened by notch (Lazzarin and Tovo, 1996, Savruk and Kazberuk, 2006-2014, Savruk et al., 2012) is a necessary step in the prediction of their strength and safety. Since these structural elements have finite dimensions or curvilinear boundary, the possibility of the application of analytical methods for solving the corresponding boundary value problems (Muskhelishvili, 2003 ) is significantly limited, and in most cases impossible.

In this paper, we provide comparison of the obtained solutions of plane elasticity problems on uniaxial loading of a plate structural element with notch of an arbitrary smooth contour by analytic method using the complex Kolosov-Muskhelishvili potentials (Kuz, 2005) and numerical method on base of the variation-difference method (Kuz, 2008, Kuz et al., 2014).

\section{ANALYTIC METHOD FOR SOLVING THE PROBLEM}

Let us find the stress state of a plate of the thicknessh, which is simulated by the half-plane, on the surface of which a notch is made of an arbitrary smooth contour. We assume that the halfplane extends to infinity by normal stress of value $P$ (Fig. 1), and the boundary of the half-plane with notch is free from stresses.

Choose a Cartesian coordinate system $O x y$, directing the axis $O x$ along the straight edge, and the vertical axis - upwards. The curve traced by the notch is denoted by $L$, the straight line portionof the boundary of the half-plane by $L^{\prime}$. The lower halfplane of the plane $x O y$ is denoted by $S^{-}$, the upper one by $S^{+}$.

According to the formulation of the problem we have the following boundary conditions:

$\Sigma_{y y}=0, \quad \Sigma_{x y}=0, \quad x \in L^{\prime} ; N=T=0, \quad t \in L$, where $N$ and $T$ are the normal and tangential components of the vector of stresses on $L$ respectively.

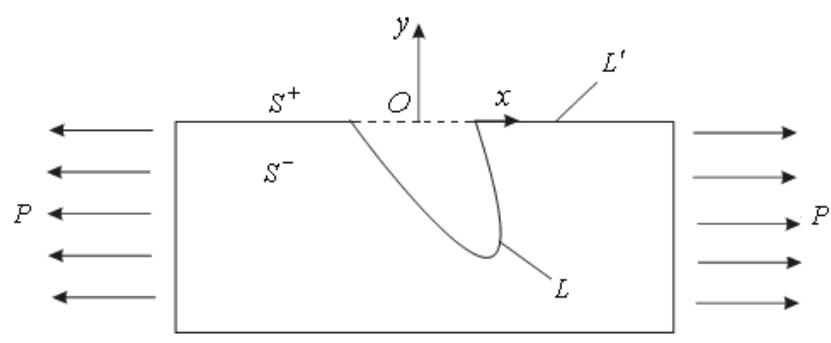

Fig. 1. Plate with the notch under uniaxial loading

To solve the problem, we introduce the complex KolosovMuskhelishvili potentials $\Phi(\mathrm{z})$ and $\Psi(\mathrm{z})$ and present them in the form:

$$
\Phi(z)=\Phi_{1}(z)+\Phi_{2}(z)+\frac{p}{4}, \quad \Psi(z)=\Psi_{1}(z)+\Psi_{2}(z)-\frac{p}{2} .
$$

Here $\Phi_{2}(z)$ and $\Psi_{2}(z)$ are complex potentials which are holomorphic in the lower half-plane and must ensure that the zero boundary conditions on the axis $y=0$, are fulfilled, and corrective complex potentials $\Phi_{1}(z)$ and $\Psi_{1}(z)$ are responsible for the implementation of the boundary conditions on the surface of the notch.

Analytically extending the function $\Phi_{2}(z)$ from the region $S^{-}$ over the region $S^{+}$and solving the corresponding problem of conjugation of boundary values on the line, we obtain a singular integral equation, which we solve numerically using the method of mechanical quadratures (Panasiuk et al., 1976). 


\section{VARIATION-DIFFERENCE METHOD FOR SOLVING THE PROBLEM}

We also consider the plane problem of elasticity theory in a finite region $V$ with curved boundary $\Sigma$ (see Fig. 1), which simulates the stress-strain state in a plate with notch of an arbitrary smooth contour. From the mathematical point of view it consists in solving equations of equilibrium in a plate (Bozydarnik and Sulym, 2012):

$\left(C_{i j k l} u_{k, l}\right)_{, j}=0$

using mixed boundary conditions on the surface $\Sigma$

$\left.C_{i j k l} u_{k, l} n_{j} \quad\right|_{\Sigma}=P_{i}$.

Here $C_{i j k l}$ are the components of the elastic modulus tensor; $u_{i}, P_{i}, n_{j}$ are the components of the displacement vector, surface forces, and the external normal to the surface $\Sigma$ respectively; $u_{i, j} \equiv \partial u_{i} / \partial x_{j}$. We assume the summation from one to two by the same indices that occur twice in one expression.

For numerical solution of problem (1) - (2) it is convenient to use its variation formulation (Pobedria, 1981), which is to minimize the Lagrangian:

$L=\int_{V} W d V-\int_{\Sigma} P_{i} u_{i} d \Sigma$,

where $W=\frac{1}{2} C_{i j k l} u_{i, j} u_{k, l}$ is the energy density of elastic deformation.
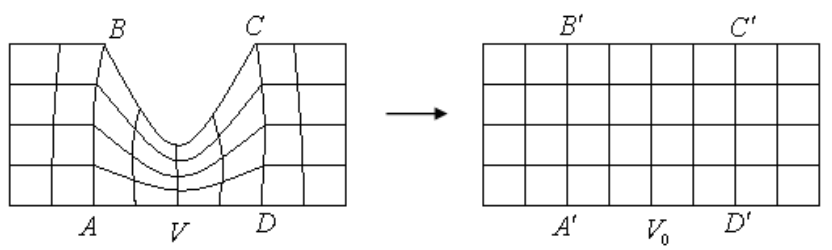

Fig. 2. Mapping of a grid in the curvilinear region $V$ onto the uniform rectangular grid in the region $V_{0}$

We write the Lagrangian (3) in the canonical region $V_{0}$, which can be a rectangle or a region composed of rectangles. For this purpose we use a discrete bijection mapping of the grid in a curvilinear region $V$ to a uniform rectangular grid $N_{1} \times N_{2}$ of the region $V_{0}$ (Fig. 2):

$x_{i}=x_{i}\left(\beta^{1}, \beta^{2}\right) \quad(i=1,2)$.

Then $J=\operatorname{det}\left(A_{i}^{j}\right), g_{i j}=A_{i}^{m} A_{j}^{m}$, where $A_{i}^{j}=\partial x_{i} / \partial \beta^{j}$ is the Jacobi matrix of this mapping. Using (4) we write the density energy of the deformation Win the coordinates $\vec{\beta}$

$$
\begin{gathered}
W=\frac{1}{2} C^{i j k l} u_{i, j} u_{k, l} \\
=\frac{1}{2} C^{i j k l}(\vec{\beta}) B_{j}^{m} B_{l}^{n} u_{i \mid m} u_{k \mid n} \\
=\frac{1}{2} D^{i m k n}(\vec{\beta}) \mathrm{u}_{\mathrm{i} \mid \mathrm{m}} \mathrm{u}_{\mathrm{k} \mid \mathrm{n}}
\end{gathered}
$$

where $\quad u_{i \mid m} \equiv \partial u_{i} / \partial \beta^{m}, \quad B_{j}^{m}=\partial \beta^{m} / \partial x_{j}, \quad D^{i m k n}=$ $C^{i j k l} B_{j}^{m} B_{l}^{n}$.

Thus, the Lagrangian in the rectangle $V_{0}$ will look like:
$L_{0}=\frac{1}{2} \int_{V_{0}} J D^{i m k n} u_{i \mid m} u_{k \mid n} d v-\int_{\Sigma_{0}} q(\vec{\beta}) P_{i} u_{i} d \Sigma$,

where $q(\vec{\beta})= \begin{cases}\sqrt{g_{11}}, & \beta^{2}=\left\{0, l_{2}\right\}, \\ \sqrt{g_{22}}, & \beta^{1}=\left\{0, l_{1}\right\} .\end{cases}$

Replacing in (5) all continual function by grid ones, integrals by finite sums, and derivatives by difference derivatives, we obtain the difference analogue of the Lagrangian $L_{0}^{h}$ using the discrete analogue of mapping (4), which should not be given analytically, in particular, to be conformal. It is sufficient to have one correspondence between nodes in the curvilinear $V_{1}$ and model $V_{0}$ regions. To determine the stationary point $L_{0}^{h}$ we obtain a system of linear algebraic equations:

$\partial L^{h} / \partial v_{\beta}^{h}\left(i_{1}, i_{2}\right)=0, \quad i_{\alpha}=1,2, \ldots, N_{\alpha}, \quad \alpha, \beta=1,2$.

This approach leads to the impossibility of the use of direct methods for solving the system (6) due to the accumulation of errors of rounding. However, it was done with a combined iterative process that implements the scheme of the gradient method and the method with Chebyshev set of iterative parameters. The complexity of its practical implementation is selection of iterative parameters.

The described variation-difference method in domains with curved boundary is implemented as a software on FORTRAN.

\section{RESULTS}

For example, the calculations of the components of the stress tensor on the notch and near it done, if its boundary is an arc of the circle, ellipse or parabola.

In Fig. 3 and Fig. 4 there are shown the graphs of dimensionless stresses $\sigma_{\theta \theta}^{0} \equiv \sigma_{\theta \theta} / P, \sigma_{x x}^{0} \equiv \sigma_{x x} / P$ and $\sigma_{y y}^{0} \equiv \sigma_{y y} / P$ for the notches along the arc of the circle. Here and after, 2lis the width of the notch (along the axis Ox); $\delta$ is the depth of the notch (along the axis Oy); $a=\delta / l$ is a dimensionless parameter relative absorption; $\sigma_{\theta \theta}^{0}$ is dimensionless circumferential stress on the notch, $\sigma_{x x}^{0}, \sigma_{y y}^{0}$ are dimensionless normal stresses on a segment $x^{0} \equiv x / l=0$, $y^{0} \equiv y / \delta \in[-5,-1]$ (along the axis Oy below the groove). The hatched lines represent stress obtained by the analytic method, and the solid lines by variation-difference method.

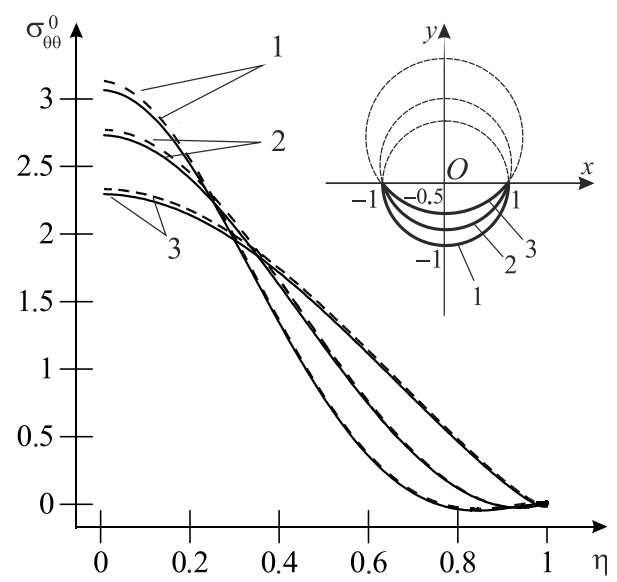

Fig. 3. Stress $\sigma_{\theta \theta}^{0}$ on the notch in an arc of the circle for $l=1$ at different values of $\delta$ 
In Fig. 3, the curves 1 are obtained for $\delta=1$, curves 2 for $\delta=0.75$, curves 3 for $\delta=0.5$. As seen from this figure, the stress $\sigma_{\theta \theta}^{0}$ (actually the coefficient of stress concentration) in the top of the notch $(\eta=0)$ achieves its greatest value in the case of notch along the semicircle (curve 1). And it is only slightly higher than typical for the Kirsch problem value 3.

Here and after, the accuracy of the results is of four significant digits (the error of about $0,1 \%$ ). Monitoring convergence and accuracy of analytic and numerical solution is conducted by comparing the studied parameters on the grids with single and double number of nodes.
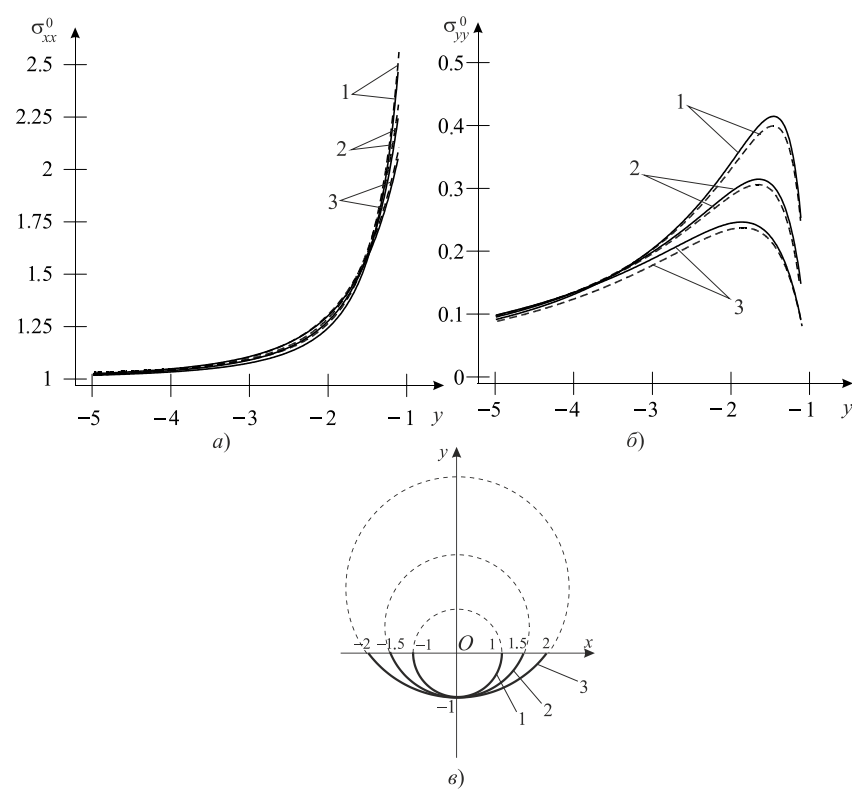

Fig. 4. Stresses $\sigma_{x x}^{0}$ and $\sigma_{y y}^{0}$ on extension of the axis of symmetry of the notch along the arc of a circle $\delta=1$ for various values of $l$

In Fig. 4, the curves 1 are obtained for $l=1$, curves 2 for $l=1.5$, curves 3 for $l=2$. As shown in Fig. 4 , the normal stress $\sigma_{\mathrm{xx}}^{0}$ much lower of the notch $(\mathrm{y}=-5)$ is almost equal $P$, and at the top of the notch it is obvious that $\sigma_{x x}^{0}=\sigma_{\theta \theta}^{0}$.

Fig. 5 and Fig. 6 show the relevant graphs of stresses $\sigma_{\theta \theta}^{0}, \quad \sigma_{x x}^{0}$ and $\sigma_{y y}^{0}$ for the notches along the arc of the ellipse.

In Fig. 5 the curves 1 are obtained for $\delta=0.5$, curves 2 for $\delta=0.75$, curves 3 for $\delta=1$, curves 4 for $\delta=1.5$.

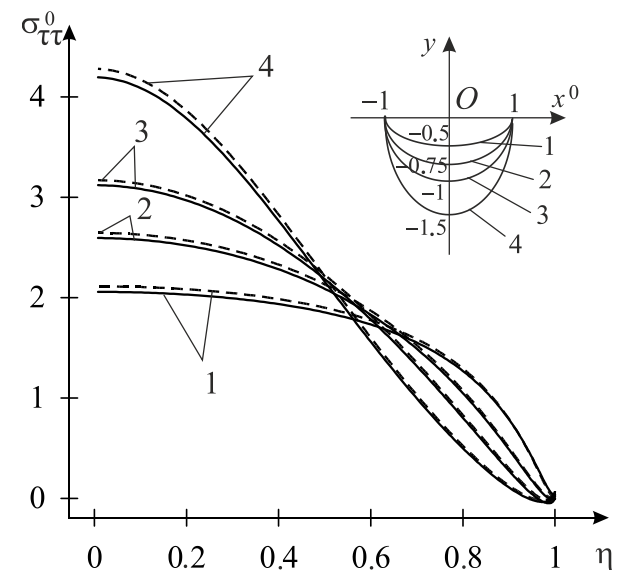

Fig. 5. Stress $\sigma_{\tau \tau}^{0}$ on the notch along the arc of the ellipse for $l=1$ and various values of $\delta$

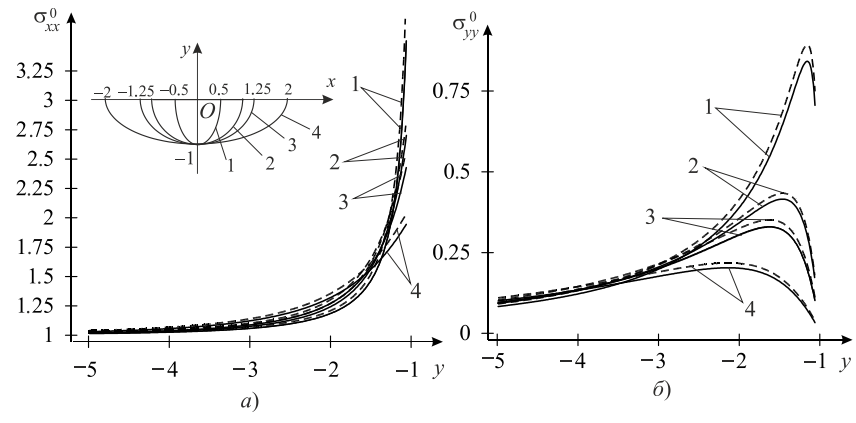

Fig. 6. Stresses $\sigma_{x x}^{0}$ and $\sigma_{y y}^{0}$ on the extension of the symmetry axis of the notch along the arc of the ellipse for $\delta=1$ and various values of $l$

In Fig. 6, the curves 1 are obtained for $l=0.5$, curves 2 for $l=1$, curves 3 for $l=1.25$, curves 4 forl $=2$.

Fig. 7 and Fig. 8 show the relevant graphs of stresses $\sigma_{\tau \tau}^{0}, \quad \sigma_{\mathrm{xx}}^{0}$ and $\sigma_{\mathrm{yy}}^{0}$ for the notches along the arc of the parabola.

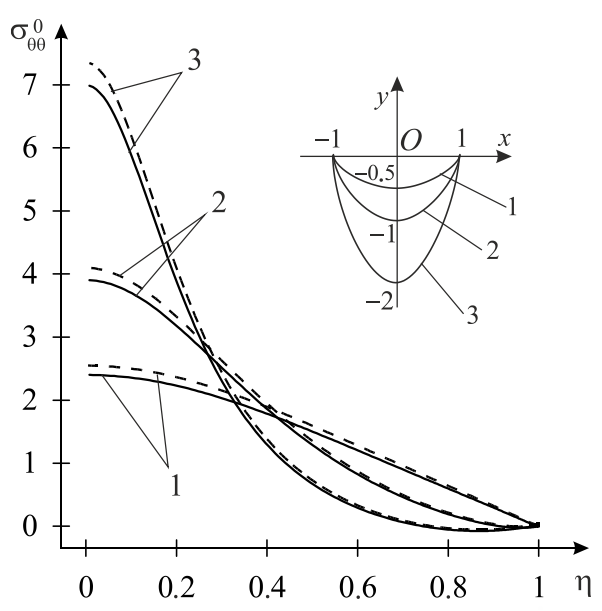

Fig. 7. Stress $\sigma_{\tau \tau}^{0}$ on the notch along the arc of the parabola for $l=1$ and various values of $\delta$

In Fig. 7, the curves 1 are obtained for $\delta=0.5$, curves 2 for $\delta=1$, curves 3 for $\delta=2$.
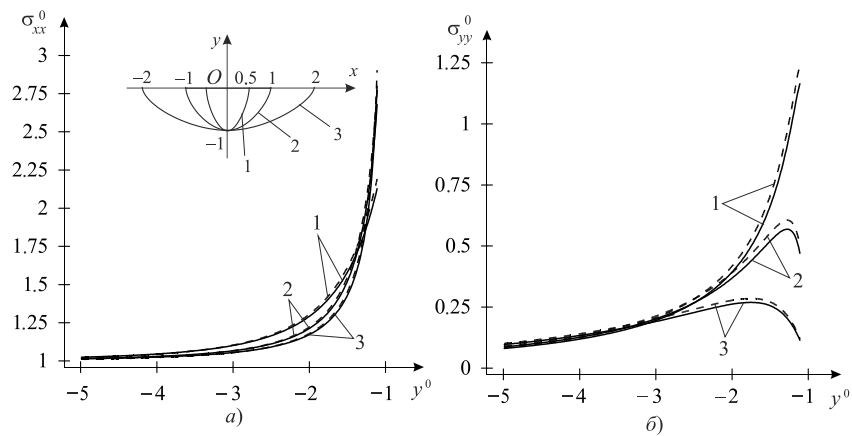

Fig. 8. Stresses $\sigma_{x x}^{0}$ and $\sigma_{y y}^{0}$ on the extension of the symmetry axis of the notch along the arc of the parabola for $\delta=1$ and various values of $l$

In Fig. 8, the curves 1 are obtained for $l=0.5$, curves 2 for $l=1$, curves 3 for $l=2$. 
Fig. 9 shows the change of the stress $\sigma_{\tau \tau}^{0}$ for the three types of notches of the same depth (arces of circle, parabola and ellipse that pass through three fixed points), which enables us to identify the influence of notch shape on the stress state of the plate.

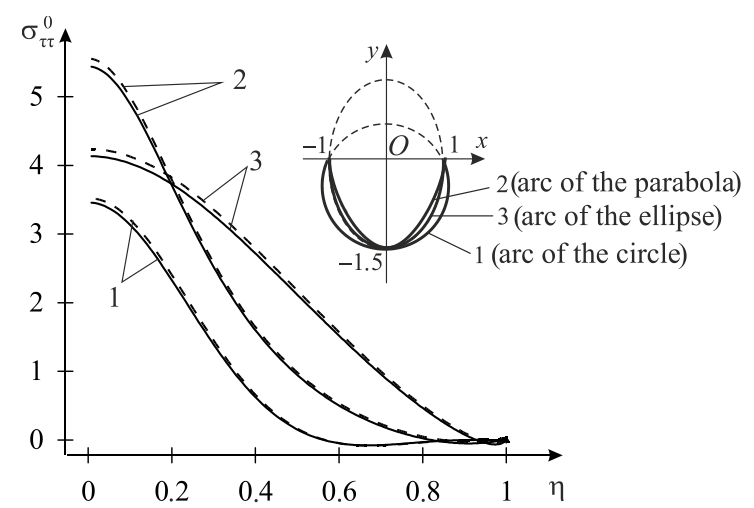

Fig. 9. Stress $\sigma_{\tau \tau}^{0}$ on the surface of the notches of the same depth along the arc of the circle, parabola, and ellipse for $l=1, \delta=1.5$

In Fig. 9, curve 1 concerns the circle, curve 2 the parabola, and curve 3 the ellipse.

\section{CONCLUSIONS}

As is shown in Fig. 5-8, the given stresses at the top of the notch along the arc of an ellipse or a parabola significantly increase with increasing of the relative depth of the notch (while increasing its depth or decreasing width). As is shown in Fig. 9, sharpness of the notch, obviously, also enlarges the level of stresses.

As is shown in Fig. 3-9, the stress fields obtained by analytic and variation-difference methods differ by no more than $2 \%$. This discrepancy can be explained by the fact that in the analytical solution domain is unbounded, while the numerical calculation was carried out, obviously, for a finite field.

Thus, the developed method of numerical determination of stresses and their concentrations agrees at solving plane elasticity problems in the plates with notch.

\section{REFERENCES}

1. Bozydarnik V. V., Sulym H. T. (2012), Theory of Elasticity, V. 1, Publishing House of Lutsk National Technical University, Lutsk (in Ukrainian).

2. Kuz I. (2008), Numerical solution of plane plasticity problem about metal elbow strain, Visnyk of Lviv University, Ser. Mech. et Math., 69, 203-209 (in Ukrainian).

3. Kuz I., Kuz O., Pyz N. (2014), Influence of stress concentrators onto stress-strain state of elasto-plastic plates, Visnyk of the Ternopil National Technical University, 4(76), 79-88 (in Ukrainian).

4. Kuz O. (2005), Stress state of semi-plane with notch under uniform extension, Abstract of the Sixth Polish-Ukrainian Conference "Current problems of mechanics of nonhomogeneous media", Warsaw, 76-77 (in Ukrainian).

5. Lazzarin P., Tovo R. (1996), A unified approach to the evaluation of linear elastic stress fields in the neibourhood of cracks or notches, International Journal of Fracture, 78, 3-19.

6. Muskhelishvili N.I. (2003), Some Basic Problems of the Mathematical Theory of Elasticity, Springer.

7. Panasiuk V. V., Savruk M. P., Datsyshyn O. P. (1976), Distribution of Stresses near Cracks in Plates and Shells, Naukova Dumka, Kyiv (in Russian).

8. Pobedria B. E. (1981), Numerical Methods in Theory of Elasticity and Plasticity, Publishing House of Moscow University, Moscow (in Russian).

9. Savruk M.P., Kazberuk A. (2006), Relationship between the stress intensity and stress concentration factor for sharp and rounded notches, Material Science (Springer), 42(6), 725-738.

10. Savruk M.P., Kazberuk A. (2007), A unified approach to problems of stress concentration near $V$-shaped notches with sharp and rounded tip, International Applied Mechanics, 43(2), 182-187.

11. Savruk M.P., Kazberuk A. (2007), Stress concentration near a rounded v-notch with arbitrary vertex curvature, Acta mechanica et Automatica, 1(1), 99-102 (in Polish).

12. Savruk M.P., Kazberuk A. (2010), Two-dimensional fracture mechanics problems for solids with sharp and rounded V-notches, International Journal of Fracture, 161, 79-95.

13. Savruk M.P., Kazberuk A. (2011), Antisymmetric stress distribution in an elastic body with a sharp or a rounded v-shaped notch, Material Science (Springer), 46(6), 711-722.

14. Savruk M.P., Kazberuk A. (2012), Distribution of stresses near Vshaped notches in the complex stressed state, Material Science (Springer), 47(4), 476-487.

15. Savruk M.P., Kazberuk A. (2014), Curvilinear cracks in the anisotropic plane and the limit transition to the degenerate material, Material Science (Springer), 50(2), 189-200.

16. Savruk M.P., Kazberuk A. (2014), Plane elgenvalue problems of the elasticity theory for orthotropic and quasi-orthotropic wedges, Material Science (Springer), 50(6), 707-714.

17. Savruk M. P., Kazberuk A., Tarasiuk G. (2012), Distribution of stresses over the contour of rounded V-shaped notch under antiplane deformation, Material Science (Springer), 47(6), 717-725. 